\title{
Collaborative Software Development Using R-Forge
}

\author{
Special invited paper on "The Future of $R^{\text {" }}$ \\ by Stefan Theußl and Achim Zeileis
}

\section{Introduction}

Open source software (OSS) is typically created in a decentralized self-organizing process by a community of developers having the same or similar interests (see the famous essay by Raymond, 1999). A key factor for the success of OSS over the last two decades is the Internet: Developers who rarely meet face-to-face can employ new means of communication, both for rapidly writing and deploying software (in the spirit of Linus Torvald's "release early, release often paradigm"). Therefore, many tools emerged that assist a collaborative software development process, including in particular tools for source code management (SCM) and version control.

In the R world, SCM is not a new idea; in fact, the $\mathrm{R}$ Development Core Team has always been using SCM tools for the R sources, first by means of Concurrent Versions System (CVS, see Cederqvist et al., 2006), and then via Subversion (SVN, see Pilato et al., 2004). A central repository is hosted by ETH Zürich mainly for managing the development of the base R system. Mailing lists like R-help, R-devel and many others are currently the main communication channels in the R community.

Also beyond the base system, many $R$ contributors employ SCM tools for managing their $\mathrm{R}$ packages, e.g., via web-based SVN repositories like SourceForge (http://SourceForge.net/) or Google Code (http://Code.Google.com/). However, there has been no central SCM repository providing services suited to the specific needs of $R$ package developers. Since early 2007, the R-project offers such a central platform to the $\mathrm{R}$ community. R-Forge (http://R-Forge.R-project.org/) provides a set of tools for source code management and various webbased features. It aims to provide a platform for collaborative development of $\mathrm{R}$ packages, R-related software or further projects. R-Forge is closely related to the most famous of such platforms-the world's largest OSS development website-namely http: //SourceForge. net/.

The remainder of this article is organized as follows. First, we present the core features that RForge offers to the $\mathrm{R}$ community. Second, we give a hands-on tutorial on how users and developers can get started with R-Forge. In particular, we illustrate how people can register, set up new projects, use RForge's SCM facilities, provide their packages on R-
Forge, host a project-specific website, and how package maintainers submit a package to the Comprehensive R Archive Network (CRAN, http://CRAN. R-project.org/). Finally, we summarize recent developments and give a brief outlook to future work.

\section{R-Forge}

R-Forge offers a central platform for the development of $\mathrm{R}$ packages, R-related software and other projects.

R-Forge is based on GForge (Copeland et al., 2006) which is an open source fork of the 2.61 SourceForge code maintained by Tim Perdue, one of the original SourceForge authors. GForge has been modified to provide additional features for the R community, namely a CRAN-style repository for hosting development releases of $\mathrm{R}$ packages as well as a quality management system similar to that of CRAN. Packages hosted on R-Forge are provided in source form as well as in binary form for Mac OS X and Windows. They can be downloaded from the website of the corresponding project on R-Forge or installed directly in R; for a package foo, say, install.packages ("foo", repos = "http://R-Forge.R-project.org").

On R-Forge, developers organize their work in so-called "Projects". Every project has various tools and web-based features for software development, communcation and other services. All features mentioned in the following sections are accessible via socalled "Tabs": e.g., user accounts can be managed in the My Page tab or a list of available projects can be displayed using the Project Tree tab.

Since starting the platform in early 2007, more and more interested users registered their projects on R-Forge. Now, after more than two years of development and testing, around 350 projects and more than 900 users are registered on R-Forge. This and the steadily growing list of feature requests show that there is a high demand for centralized source code management tools and for releasing prototype code frequently among the R community.

In the next three sections, we summarize the core features of R-Forge and what R-Forge offers to the $\mathrm{R}$ community in terms of collaborative development of R-related software projects.

\section{Source code management}

When carrying out software projects, source files change over time, new files get created and old files deleted. Typically, several authors work on several computers on the same and/or different files and 
keeping track of every change can become a tedious task. In the open source community, the general solution to this problem is to use version control, typically provided by the majority of SCM tools. For this reason R-Forge utilizes SVN to facilitate the developer's work when creating software.

A central repository ensures that the developer always has access to the current version of the project's source code. Any of the authorized collaborators can "check out" (i.e., download) or "update" the project file structure, make the necessary changes or additions, delete files from the current revision and finally "commit" changes or additions to the repository. More than that, SVN keeps track of the complete history of the project file structure. At any point in the development stage it is possible to go back to any previous stage in the history to inspect and restore old files. This is called version control, as every stage automatically is assigned a unique version number which increases over time.

On R-Forge such a version-controlled repository is automatically created for each project. To get started, the project members just have to install the client of their choice (e.g., Tortoise SVN on Windows or svnX on Mac OS X) and check out the repository. In addition to the inherent backup of every version within the repository a backup of the whole repository is generated daily.

A rights management system assures that, by default, anonymous users have read access and developers have write access to the data associated with a certain project on R-Forge. More precisely, registered users can be granted one of several roles: e.g., the "Administrator" has all rights including the right to add new users to the project or release packages directly to CRAN. He/she is usually the package maintainer, the project leader or has registered the project originally. Other members of a project typically have either the role "Senior Developer" or "Junior Developer" which both have permission to commit to the project SVN repository and examine the log files in the $R$ Packages tab (the differences between the two roles are subtle, e.g., senior developers additionally have administrative privileges in several places in the project). When we speak of developers in subsequent sections we refer to project members having the rights at least of a junior developer.

\section{Release and quality management}

Development versions of a software project are typically prototypes and are subject to many changes. Thus, R-Forge offers two tools which assist the developers in improving the quality of their source code.

First, it offers a quality management system similar to that of CRAN. Packages on R-Forge are checked in a standardized way on different platforms based on R CMD check at least once daily. The resulting log files can be examined by the project de- velopers so that they can improve the package to pass all tests on R-Forge and subsequently on CRAN.

Second, bug tracking systems allow users to notify package authors about problems they encounter. In the spirit of OSS-given enough eyeballs, all bugs are shallow (Raymond, 1999) - peer code review leads to an overall improvement of the quality of software projects.

\section{Additional features}

A number of further tools, of increased interest for larger projects, help developers to coordinate their work and to communicate with their user base. These tools include:

- Project websites: Developers may present their work on a subdomain of R-Forge, e.g., http: //foo.R-Forge.R-project.org/, or via a link to an external website.

- Mailing lists: By default a list foo-commits lists.R-Forge.R-project.org is automatically created for each project. Additional mailing lists can be set up easily.

- Project categorization: Administrators may categorize their project in several so-called "Trove Categories" in the Admin tab of their project (under Trove Categorization). For each category three different items can be selected. This enables users to quickly find what they are looking for using the Project Tree tab.

- News: Announcements and other information related to a project can be put on the project summary page as well as on the home page of R-Forge. The latter needs approval by one of the R-Forge administrators. All items are available as RSS feeds.

- Forums: Discussion forums can be set up separately by the project administrators.

\section{How to get started}

This section is intended to be a hands-on tutorial for new users. Depending on familiarity with the systems/tools involved the instructions might be somewhat brief. In case of problems we recommend consulting the user's manual (R-Forge Administration and Development Team, 2008) which contains detailed step-by-step instructions.

When accessing the URL http://R-Forge. $\mathrm{R}$-project.org/ the home page is presented (see Figure 1). Here one can

- login,

- register a user or a project, 


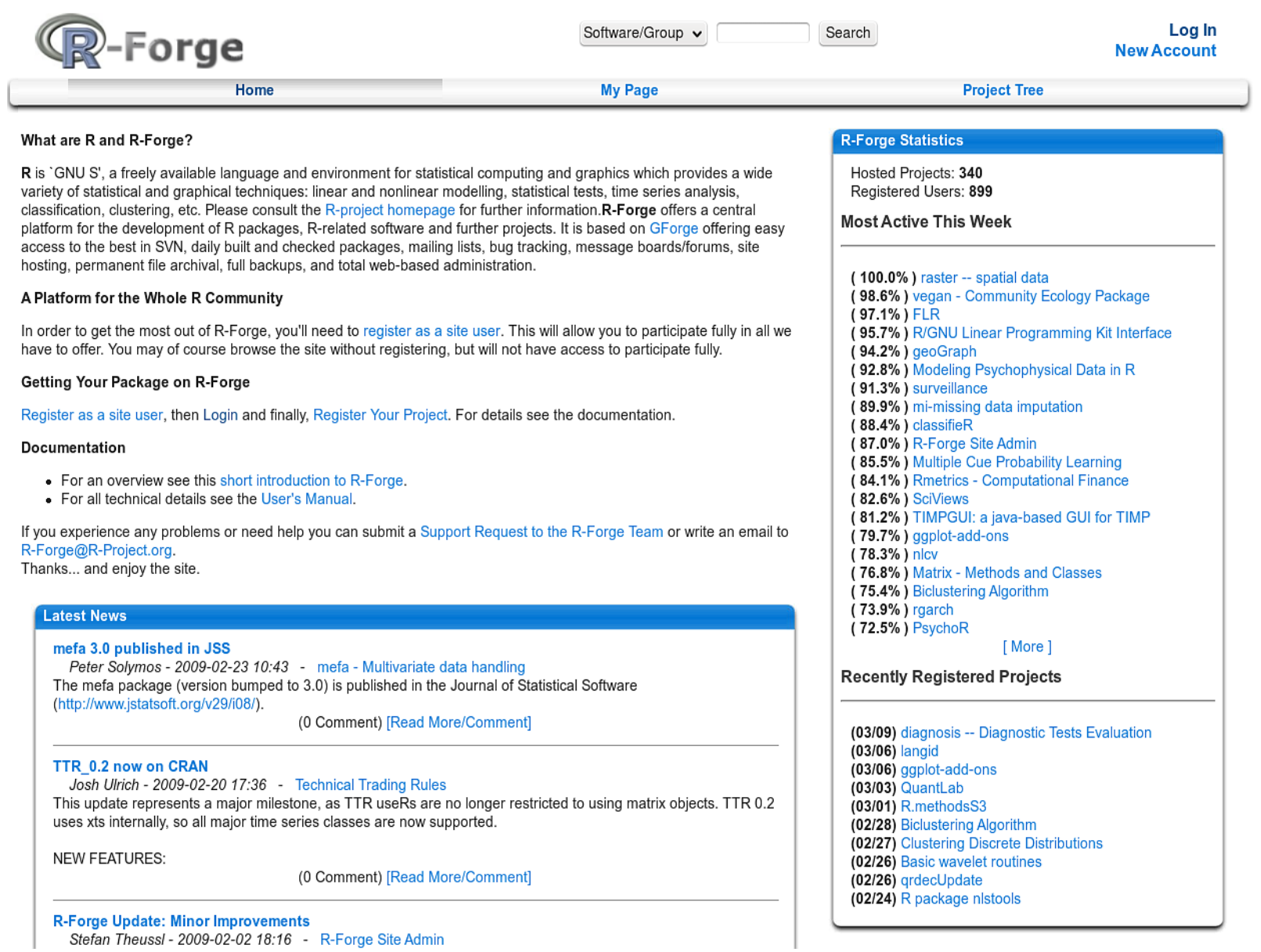

Figure 1: Home page of R-Forge on 2009-03-10

- download the documentation,

- examine the latest news and changes,

- go to a specific project website either by searching for available projects (top middle of the page), by clicking on one of the projects listed on the right, or by going through the listing in the Project Tree tab.

Registered users can access their personal page via the tab named My Page. Figure 2 shows the personal page of the first author.

\section{Registering as a new user}

To use R-Forge as a developer, one has to register as a site user. A link on the main web site called New Account on the top right of the home page leads to the corresponding registration form.

After submitting the completed form, an e-mail is sent to the given address containing a link for activating the account. Subsequently, all R-Forge features, including joining existing or creating new projects, are available to logged-in users.

\section{Registering a project}

There are two possibilities to register a project: Clicking on Register Your Project on the home page or going to the My Page tab and clicking on Register Project (see Figure 2 below the main tabs). Both links lead to a form which has to be filled out in order to finish the registration process. In the text field "Project Public Description" the registrant is supposed to enter a concise description of the project. This text and the "Project Full Name" will be shown in several places on R-Forge (see e.g., Figure 1). The text entered in the field "Project Purpose And Summarization" is additional information for the R-Forge administrators, inspected for approval. "Project Unix Name" refers to the name which uniquely determines the project. In the case of a project that contains a single $\mathrm{R}$ package, the project Unix name typically corresponds to the package name (in its lower-case version). Restrictions according to the Unix file system convention force Unix names to be in lower case (and will be converted automatically if they are typed in upper case).

After the completed form is submitted, the project has to be approved by the R-Forge administrators and a confirmation e-mail is sent to the registrant upon approval. After that, the regis- 


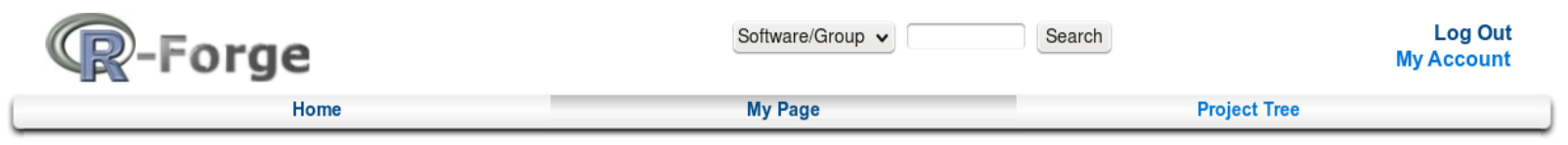

My Personal Page | Diary \& Notes | Account Maintenance | Register Project

Your personal page contains lists of bugs and tasks that you are assigned, plus a list of groups that you are a member of.

\begin{tabular}{|c|c|}
\hline My As & ned Items \\
\hline R-FC & Site Admin - Support \\
\hline 180 & Ability to rename project? \\
\hline 154 & package checking options \\
\hline R-FC & Site Admin - Feature Requests \\
\hline 99 & Automatic case conversion for folder names under Windows XP \\
\hline 65 & Prominent place for additional project information \\
\hline 64 & ([Semi-] automatic) maintenance of a target / todo list \\
\hline 16 & automated R.check delivery \\
\hline My S & nitted Items \\
\hline R-F & Site Admin - Feature Requests \\
\hline 17 & R-Forge package download statistic \\
\hline 16 & automated R.check delivery \\
\hline Moni & d Forums \\
\hline R-F & Site Admin \\
\hline 8 & elopers \\
\hline Moni & d FileModules \\
\hline You & not monitoring any files. \\
\hline $\begin{array}{l}\text { If yo } \\
\text { new }\end{array}$ & $\begin{array}{l}\text { onitor files, you will be sent new release notices via email, with a link to the } \\
\text { on our download server. }\end{array}$ \\
\hline $\begin{array}{l}\text { You } \\
\text { chec }\end{array}$ & $\begin{array}{l}\text { monitor files by visiting a project's "Summary Page" and clicking on the } \\
\text { ox in the files section. }\end{array}$ \\
\hline
\end{tabular}

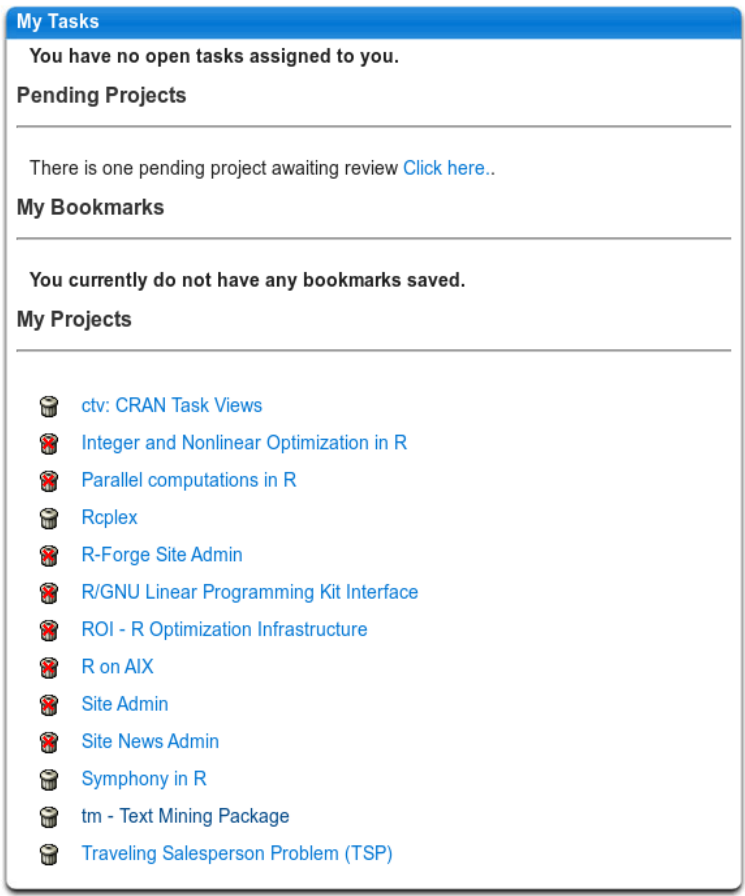

Figure 2: The My Page tab of the first author

trant automatically becomes the project administrator and the standardized web area of the project (http://R-Forge.R-project.org/projects/foo/) is immediately available on R-Forge. This web area includes a Summary page, an Admin tab visible only to the project administrators, and various other tabs depending on the features enabled for this project in the Admin tab. To present the new project to a broader community the name of the project additionally is promoted on the home page under "Recently Registered Projects" (see Figure 1).

Furthermore, within an hour after approval a default mailing list and the project's SVN repository containing a 'README' file and two pre-defined directories called ' $p k$ ' $^{\prime}$ and ' $w w w$ ' are created. The content of these directories is used by the R-Forge server for creating $\mathrm{R}$ packages and a project website, respectively.

\section{SCM and R packages}

The first step after creation of a project is typically to start generation of content for one (or more) $R$ package(s) in the 'pkg' directory. Developers can either start committing changes via SVN as usual or- if the package is already version-controlled somewhere else-the corresponding parts of the repository including the history can be migrated to R-Forge (see Section 6 of the user's manual).

The SCM tab of a project explains how the corresponding SVN repository located at svn://svn.R-Forge.R-project.org/svnroot/foo

can be checked out. From this URL the sources are checked out either anonymously without write permission (enabled by default) or as developer using an encrypted authentication method, namely secure shell (ssh). Via this secure protocol (svn+ssh:// followed by the registered user name) developers have full access to the repository. Typically, the developer is authenticated via the registered password but it is also possible to upload a secure shell key (updated hourly) to make use of public/private key authentication. Section 3.2 of the user's manual explains this process in more detail.

To make use of the package builds and checks the package source code has to be put into the 'pkg' directory of the repository (i.e., 'pkg/DESCRIPTION', 'pkg/R', 'pkg/man', etc.) or, alternatively, a subdirectory of ' $\mathrm{pkg}$ '. The latter structure allows developers to have more than one package in a single project; e.g., 
if a project consists of the packages foo and bar, then the source code is located in 'pkg/foo' and 'pkg/bar', respectively.

R-Forge automatically examines the 'pkg' directory of every repository and builds the package sources as well as the package binaries on a daily basis for Mac OS X and Windows (if applicable). The package builds are provided in the $R$ Packages tab (see Figure 3) for download or can be installed directly in R using install.packages ( foo", repos="http: //R-Forge.R-project .org") . Furthermore, in the $R$ Packages tab developers can examine logs of the build and check process on different platforms.

To release a package to CRAN the project administrator clicks on Submit this package to CRAN in the $R$ Packages tab. Upon confirmation, a message will be sent to CRANQR-project.org and the latest successfully built source package (the '.tar.gz' file) is automatically copied to ftp://CRAN.R-project. org/incoming/. Note that packages are built once daily, i.e., the latest source package does not include more recent code committed to the SVN repository.

Figure 3 shows the $R$ Packages tab of the $\mathbf{t m}$ project (http://R-Forge.R-project.org/projects/ $\mathrm{tm} /$ ) as one of the project administrators would see it. Depending on whether you are a member of the project or not and your rights you will see only parts of the information provided for each package.

\section{Further steps}

A customized project website, accessible through http://foo.R-Forge.R-project.org/ where foo corresponds to the unix name of the project, is managed via the ' $w w w$ ' directory. The website gets updated every hour.

The changes made to the project can be examined by entering the corresponding standardized web area. On entry, the Summary page is shown. Here, one can

- examine the details of the project including a short description and a listing of the administrators and developers,

- follow a link leading to the project homepage,

- examine the latest news announcements (if available),

- go to other sections of the project like Forums, Tracker, Lists, R Packages, etc.

- follow the download link leading directly to the available packages of the project (i.e., the $R$ Packages tab).

Furthermore, meta-information about a project can be supplied in the Admin tab via so-called "Trove Categorization".

\section{Recent and future developments}

In this section, we briefly summarize the major changes and updates to the R-Forge system during the last few months and give an outlook to future developments.

Recently added features and major changes include:

- New defaults for freshly registered projects: Only the tabs Lists, SCM and R packages are enabled initially. Forums, Tracker and News can be activated separately (in order not to overwhelm new users with too many features) using Edit Public Info in the Admin tab of the project. Experienced users can decide which features they want and activate them.

- An enhanced structure in the SVN repository allowing multiple packages in a single project (see above).

- The R package RForgeTools (Theuß1, 2009) contains platform-independent package building and quality management code used on the R-Forge servers.

- A modified News submit page offering two types of submissions: project-specific and global news. The latter needs approval by one of the R-Forge administrators (default: projectonly submission).

- Circulation of SVN commit messages can be enabled in the SCM Admin tab by project administrators. The mailing list mentioned in the text field is used for delivering the SVN commit messages (default: off).

- Mailing list search facilities provided by the Swish-e engine which can be accessed via the List tab (private lists are not included in the search index).

Further features and improvements which are currently on the wishlist or under development include

- a Wiki,

- task management facilities,

- a re-organized tracker more compatible with $\mathrm{R}$ package development and,

- an update of the underlying GForge system to its successor FusionForge (http:// FusionForge.org/).

For suggestions, problems, feature requests, and other questions regarding R-Forge please contact R-Forge@R-project .org. 


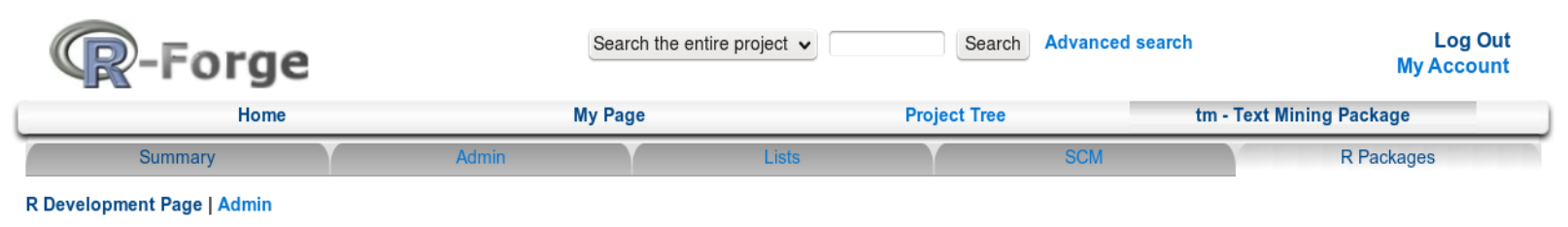

\section{Contributed R Packages}

Below is a list of all packages provided by project tm - Text Mining Package.

Important note for package binaries: R-Forge provides these binaries only for versions of $\mathrm{R}$ that were released since the package was first submitted to $\mathrm{R}$-Forge, but not for older versions. In order to use more recent packages from R-Forge you may need to switch to a newer version of R or, alternatively, try to build the package with an older version of R. Packages are built/checked according to this schedule.

\begin{tabular}{|c|c|c|c|c|}
\hline \multicolumn{5}{|c|}{ A framework for text mining applications within $\mathrm{R}$. } \\
\hline Download: $\mathrm{Pa}$ & \multicolumn{4}{|c|}{ Package source (.tar.gz) | Windows binary (.zip) | MacOS X universal binary (.tgz) } \\
\hline $\begin{array}{l}\text { Logs: } \\
\text { Daily build: } \\
\text { Daily check: }\end{array}$ & $\begin{array}{l}\text { Linux x86_32 } \\
\text { patched } \\
\text { patched | devel }\end{array}$ & $\begin{array}{l}\text { Linux x86_64 } \\
\text { patched } \\
\text { patched | devel }\end{array}$ & $\begin{array}{l}\text { Windows x86_32 } \\
\text { patched I devel } \\
\text { patched | devel }\end{array}$ & $\begin{array}{c}\text { MacOS X universal } \\
\text { patched I devel (N/A) } \\
\text { patched I devel (N/A) }\end{array}$ \\
\hline \multicolumn{5}{|c|}{ [Submit this package to CRAN] } \\
\hline \multicolumn{5}{|c|}{ To install this package directly within R type: install.packages("tm", repos="http://R-Forge.R-project.org") } \\
\hline \multicolumn{5}{|r|}{$\begin{array}{r}\text { Version: 0.3-3.2 | Last change: } 2009-03-03 \text { 17:23:22+01 | Rev.: } 894 \\
\text { Stable Release: Get tm 0.3-3 from CRAN }\end{array}$} \\
\hline
\end{tabular}

Figure 3: $R$ Packages tab of the $\mathbf{t m}$ project

\section{Acknowledgments}

Setting up this project would not have been possible without Douglas Bates and the University of Wisconsin as they provided us with a server for hosting this platform. Furthermore, the authors would like to thank the Computer Center of the Wirtschaftsuniversität Wien for their support and for providing us with additional hardware as well as a professional server infrastructure.

\section{Bibliography}

P. Cederqvist et al. Version Management with CVS. Network Theory Limited, Bristol, 2006. Full book available online at http://ximbiot.com/ $\mathrm{cvs} / \mathrm{manual} /$.

T. Copeland, R. Mas, K. McCullagh, T. Perdue, G. Smet, and R. Spisser. GForge Manual, 2006. URL http://gforge.org/gf/download/ docmanfileversion/8/1700/gforge_manual.pdf.

C. M. Pilato, B. Collins-Sussman, and B. W. Fitzpatrick. Version Control with Subversion. O'Reilly,
2004. Full book available online at http:// svnbook.red-bean.com/.

R-Forge Administration and Development Team. $R$ Forge User's Manual, 2008. URL http: // download. R-Forge.R-project.org/R-Forge.pdf.

E. S. Raymond. The Cathedral and the Bazaar. Knowledge, Technology, and Policy, 12(3):23-49, 1999.

S. Theußl. RForgeTools: R-Forge Build and Check Tools, 2009. URL http: //R-Forge. R-project. org/ projects/site/. R package version 0.3-3.

Stefan Theußl

Department of Statistics and Mathematics WU Wirtschaftsuniversität Wien

Austria

stefan.theussldwu.ac.at

Achim Zeileis

Department of Statistics and Mathematics WU Wirtschaftsuniversität Wien

Austria

achim.zeileis@wu.ac.at 